\title{
Warnings on every cigarette: extending health messaging to the consumption experience
}

\author{
Crawford Moodie PhD
}

- Cite as: CMAJ 2018 October 29;190:E1271-2. doi: 10.1503/cmaj.180781

$\mathbf{T}$ obacco use is the leading cause of premature death in Canada and costs $\$ 17$ billion in health care and indirect economic costs each year. ${ }^{1}$ Health Canada has highlighted the need for a novel, multiprong approach to reach its target of less than $5 \%$ of the population using tobacco products by $2035 .{ }^{1}$ It has dedicated $\$ 330$ million over the next five years to protecting young people and nonsmokers and helping smokers quit. ${ }^{2}$ But more must be done to reduce the appeal of tobacco further and update warning messages. One new idea, recently supported by Federal Health Minister Ginette Petitpas Taylor, is to put health warnings on individual cigarettes (Figure 1).

Some have argued that health warnings should be the first thing smokers see before buying cigarettes and the last thing they see before lighting up. ${ }^{3}$ Despite the prominence of warnings on cigarette packs in Canada, as in many other countries, some smokers avoid these using physical (covering or concealing the pack) or psychological means (ignoring warnings and focusing on branding). ${ }^{4}$ Avoidant behaviour would be more difficult with warnings on each cigarette, as smokers typically see a cigarette when taking it from a pack, when lighting it, and when it is in the ashtray. Warnings on cigarettes would extend the health message to the actual consumption experience.

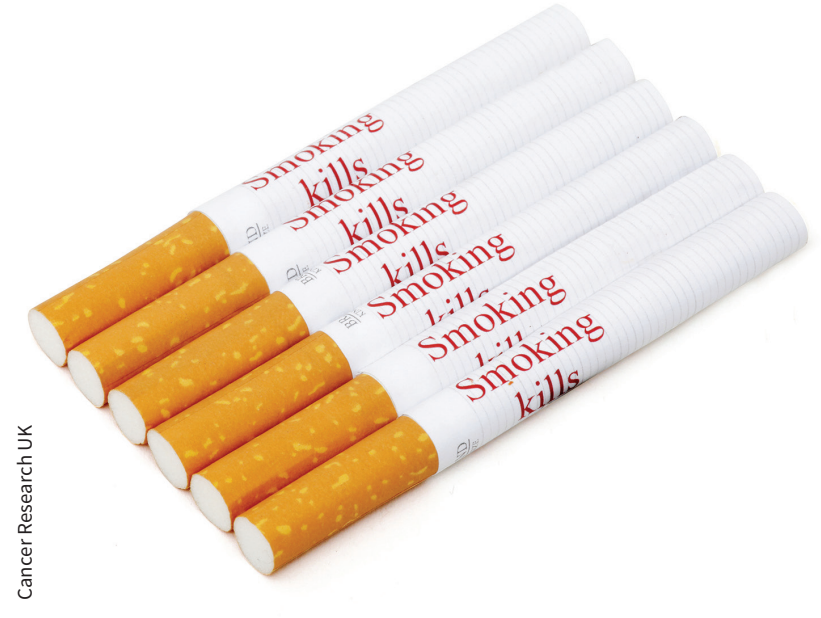

Figure 1: Warnings on each cigarette stick.

\section{KEY POINTS}

- Health Canada's tobacco control strategy recognizes a need for novel ideas to reduce smoking prevalence to less than $5 \%$ by 2035 , and the federal health minister supports the inclusion of warnings on cigarette sticks.

- Warnings on cigarettes would extend health messaging to the consumption experience and may reduce their appeal, particularly for youth, who often begin smoking with single cigarettes.

- Requiring warnings on individual cigarettes would be feasible and low cost, and may help with identifying illicit tobacco products.

- Such warnings on cigarette sticks would be consistent with past tobacco control policy, which has included warnings on cigarette packs since 1972.

Warnings on each cigarette may also be a valuable tool for reaching youth. Efforts have been made in Canada to protect youth from smoking since the Tobacco Restraint Act in 1908 made it illegal to sell cigarettes to those younger than 16 years. And yet, more than a century on, almost 1 in 10 (8.6\%) of those younger than 16 have tried smoking. ${ }^{5}$ The plain packaging amendment to the Tobacco Act is expected to have the most impact in deterring youth, but experimenters whose first smoking experience is via single cigarettes (perhaps received or stolen from friends, peers, family or others) may not be exposed to these unappealing packs when they begin smoking.

A recent meta-analysis of nationally representative surveys of adults, which found that among those who had ever tried a cigarette, more than two-thirds (68.9\%) progressed to daily smoking, highlighted the importance of reducing experimentation in youth. ${ }^{6}$ When asked for their views, marketing experts suggested that including warnings on cigarettes would ruin the image of smoking, particularly for young people. ${ }^{7}$ However, whether this would reduce experimentation is not known, as research in this area is at a nascent stage. ${ }^{8}$ In the United Kingdom, a survey of 11to 16 -year-olds ( $n=1205$ ) who were shown an image of a cigarette containing the warning "Smoking kills" found that $71 \%$ said warnings on cigarettes would put people off starting to smoke. ${ }^{9}$ However, further research is needed. 
Tobacco advertising, promotion and sponsorship - including the open display of tobacco products by retailers - is banned in Canada, and the use of plain packaging will greatly reduce the promotion of the product. Plain-packaging regulations will also standardize the appearance of cigarettes, specifically the diameter (8 $\mathrm{mm}$ ), length (71-73 mm for regular-size cigarettes, $83-85 \mathrm{~mm}$ for king-size cigarettes), filter colour (white or imitation cork) and appearance of the cigarette paper (white with an alphanumeric code in small font size). A provision could be added to require warnings to be displayed on each cigarette. As warnings have been on cigarette packs in Canada since 1972, warnings on individual cigarettes could be viewed as a logical extension of past tobacco control policy.

Printing warnings should be feasible, considering that placing branding on individual cigarettes is a long-standing practice for tobacco companies. The costs would be low and borne by the tobacco industry. Singapore set a precedent for a government requiring tobacco companies to print specific text on each cigarette. Since 2009, all cigarettes sold in Singapore must display the letters "SDPC" (Singapore Duty Paid Cigarette) as part of an effort to identify illicit cigarettes. Warnings on cigarettes in Canada could similarly help law enforcement identify illicit cigarettes.

When the Government of Canada required tobacco companies to print pictorial warnings on the outside of cigarette packs and include inserts with health messaging on the inside of packs at the start of this century, it was a world first. So it would be again if Canada legislated for warnings on individual cigarettes. Although such a move would be challenged, with every innovation in tobacco control policy having its doubters, detractors and critics, ${ }^{10}$ Canada would again be at the vanguard of tobacco control, particularly with plain packaging due to be introduced. It would be difficult to predict the impact that such a measure might have, but just as tobacco companies have adeptly exploited every element of the cigarette pack and the appearance of the cigarette to promote their products, governments could do likewise, but with the goal of promoting health and reducing the burden of tobacco-related illness on the medical profession and society.

\section{References}

1. Seizing the opportunity: the future of tobacco control in Canada. Ottawa: Health Canada; 2017. Available: www.canada.ca/content/dam/hc-sc/documents/programs/ future-tobacco-control/future-tobacco-control-consultation-eng.pdf (accessed 2018 June 6).

2. Canada's tobacco strategy. Ottawa: Health Canada; (modified 2018 June 25). Available: www.canada.ca/en/health-canada/services/publications/healthy -living/canada-tobacco-strategy.html?utm_source= canada-ca-tobacco-strategy -en\&utm_medium=vurl\&utm_campaign=vurl\#a1 (accessed 2018 Aug. 7).

3. Kaiserman MJ. The effectiveness of health warning messages. Tob Control 1993;2:267-9.

4. Maynard OM, Attwood A, O'Brien L, et al. Avoidance of cigarette pack health warnings among regular cigarette smokers. Drug Alcohol Depend 2014;136:170-4.

5. Detailed tables for the Canadian Student Tobacco, Alcohol and Drugs Survey 2016-17. Ottawa: Health Canada; 2018. Available: www.canada.ca/en/health -canada/services/canadian-student-tobacco-alcohol-drugs-survey/2016-2017 -supplementary-tables.html (accessed 2018 June 6).

6. Birge M, Duffy S, Miler JA, et al. What proportion of people who try one cigarette become daily smokers? A meta-analysis of representative surveys. Nicotine Tob Res 2017 Nov. 4 [Epub ahead of print]. doi: 10.1093/ntr/ntx243.

7. Moodie C. Novel ways of using tobacco packaging to communicate health messages: Interviews with packaging and marketing experts. Addict Res Theory 2016;24:54-61.

8. Moodie C, Gendall P, Hoek J, et al. The response of young adult smokers and non-smokers in the United Kingdom to dissuasive cigarettes: an online survey. Nicotine Tob Res 2017 Nov. 28 [Epub ahead of print]. doi: 10.1093/ntr/ntx261.

9. Moodie C, Mackintosh AM, Gallopel-Morvan K, et al. Adolescents' perceptions of health warnings on cigarettes. Nicotine Tob Res 2017;19:1232-7.

10. Malone RE. Conflicts and controversies in contemporary tobacco control. Tob Control 2017;26:e1-2.

Competing interests: Crawford Moodie reports receiving a grant (grant No. C312/A15192) from Cancer Research UK, during the conduct of the study. Cancer Research UK funds 50\% of his time until September 2018. Cancer Research UK had no involvement in this commentary.

This article has been peer reviewed.

Affiliation: Centre for Tobacco Control Research, University of Stirling, Stirling, Scotland

Correspondence to: Crawford Moodie, c.s.moodie@stir.ac.uk 